\title{
FRACTAL STABILIZATION AND STOCHASTIC MODELING OF ZINC ELECTRODEPOSITION STRUCTURES IN THIN PLATES
}

\author{
ROCIO R. GALLEGOS VILLELA, GILDARDO HERRERA SÁNCHEZ, EVANGELINA A. \\ MONTALVO-RIVERO, JOSUE F. PÉREZ-SÁNCHEZ* \& LISBETH A. BRANDT GARCÍA
}

Facultad de Arquitectura Diseño y Urbanismo, Universidad Autonoma de Tamaulipas, Tampico, Tamaulipas, Mexico

\section{ABSTRACT}

Thin-layer electrodeposition has been studied to involve complex branched morphologies, useful for different industrial processes. In this work, it was studied the electrodeposition of Zinc for a constant Zn2+ concentration and two currents, $3 \mathrm{~mA}$ and $10.3 \mathrm{~mA}$. The electrodeposition test was carried out in a thin circular layer, analyzing the variation of the massradius fractal dimension over time and the different radius of the deposits formed. The study was focused on the fractal dimension stabilization analysis and the mathematical modeling of this parameter through the stochastic methodology. It was found that the fractal dimension stabilizes depending on the radius studied and becomes constant over time. The stochastic model proposed involves the radius of the deposit and the electrodeposition time. The model predicts the fractal dimension value with a correlation of $61.43 \%$ for the highest current value, which is an indication that the faster deposition velocity produces a more stable fill-up effect of the spaces in the deposition surface.

KEYWORDS: Branched Electrodeposition; Fractal Electroplate, Zinc Reduction

Received: Aug 24, 2020; Accepted: Sep 14, 2020; Published: Nov 09, 2020; Paper Id.: IJMPERDOCT202032

\section{INTRODUCTION}

In the last decades, electrochemical processes have found several industrial applications [1-6]. Since the 1980s, it was started the study of Zinc ( $\mathrm{Zn}$ ) deposition morphology, determining that the $\mathrm{Zn}$ electrodeposition process is sensible for the experimental conditions [7-11]. For example, in a circular anode/tip cathode arrangement, where the cathode tip is centered in the anode surface, the ramifications start from the tip following the dendritical DLA mechanism (diffusion-limited aggregation) [2-7,12,13]. However, this dendritical growth is not fully explained though DLA [14]. Besides, some other structures or deposits can be found [11]: Compact (deposition with no specific structure/ramification); Homogeneous (dense ramifications with a parallel growing to cathode); Open (disordered and faltered ramifications); Assorted (dendritical/other ramifications); Needle-shaped growths without lateral ramifications.

The structures can also be modified according to the electrodeposition rate of the $\mathrm{Zn}^{2+}$ ions, which is tuned by the electric current, which in turn is lesser for a long distance between the electrodes and vice versa [14]. Some other experimental conditions can be set under constant potential or constant current, high $\mathrm{Zn}^{2+}$ concentrations and high potentials (more open structures), $\mathrm{Zn}^{2+}$ concentrations from 0.03 to $0.1 \mathrm{M}$ (usual dendritical growths like DLA) [15], electric potential ramps (uncontrolled growth) [10], and voltage oscillations [10,13]. Electrodeposition structures are ideal models for the general processes of fractal growth, and they are similar to other fractal morphology on solids [16]. Some studies focus on the time-changing fractal dimension of electrodeposition. For $0.1 \mathrm{~mm}$ diameter electrodes, with a separation of $50 \mathrm{~mm}$ and under constant potential $(12 \mathrm{~V})$, it was found a fractal 
dimension of $1.66 \pm 0.08$, and it was established that the morphologies were self-similar [8].

\section{MATERIALS AND METHODS}

To study the temporal variation of the fractal dimension was studied under two constant current values of $3 \mathrm{~mA}$ and 10.3 $\mathrm{mA}$ at $25{ }^{\circ} \mathrm{C}$, with a $\mathrm{ZnSO}_{4} 0.1026 \mathrm{M}$ (Merck, Mexico). The $\mathrm{Zn}$ growth was studied every 10 min for $30 \mathrm{~s}$ with a polarization microscope (BA310POL, Motic) at 10-40x in circular plates of acrylic crystal. The anode was annular with 44 $\mathrm{mm}$ of radius, and the anode was set up to $0.44 \mathrm{~mm}$ diameter, both electrodes were made of Pt $[2,3]$. The voltage/time variation was measured with a digital multimeter (MUL-5000, Steren). The images obtained were contrasted in black and white through the ImageJ software as in [17-19], and the diameter of the structures was measured in pixels with the Fractalyse software [18]. The fractal dimension was determined with Harfa software [19] for two cases: the final image and concentric 100 pixels thick rings. The content of $\mathrm{Zn}$ in the $\mathrm{ZnSO}_{4}$ solution was followed by titration with EDTA to determine the final concentration.

This work considers as a hypothesis that the fractal dimension is a result of the random deposition processes and the chemical reactions at the microscopic level. Thereby, the stochastic modeling of the fluctuations and the fractal geometry bases were used to estimate the fractal dimension and the system size over time. The final surface of the system is quantified from the fractal structure radius.

\section{RESULTS AND DISCUSSIONS}

Figure 1 shows the images obtained for the experiments at 3 and $10.3 \mathrm{~mA}$. For $3 \mathrm{~mA}$ tests, in most of the cases, the fractal dimension decreases per unit of time, and it is diminished while the diameter is more significant. This behavior is a result of the $\mathrm{Zn}$ random electrodeposition; thus, the empty spaces on the surface are filled with crystals reducing the ramifications at the initial stage while ramification grows 30 seconds later. The temporal growth of the mass-radium fractal dimension seems reasonable due to as the $\mathrm{Zn}$ is deposited, the area of study is increased. This behavior is also observed for the 10.3 $\mathrm{mA}$ tests, where the growth is faster. Figure 2 shows the maximum radium of the crystal growth for six samples. As it was expected, the $\mathrm{Zn}$ decay in the solution was linear, which favors the formation of $\mathrm{H}_{2}(\mathrm{~g})$. The high potential experiments were modified to be measured up to $10 \mathrm{~min}$ to avoid surface modification due to gas release.

a)
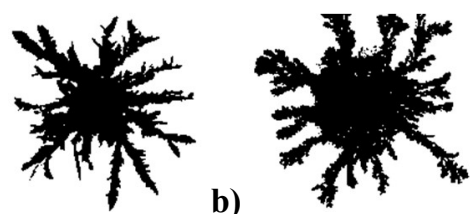

Figure 1: B/W Crystal growth fo $\mathrm{Zn}$ at a) 3 and b) $10.3 \mathrm{~mA}$.

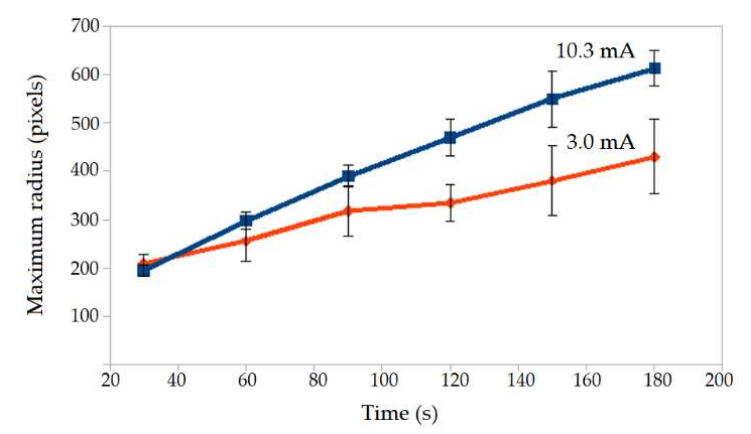

Figure 2: Maximum diameter values for 3 and $10.3 \mathrm{~mA}$. 
Figures 3 and 4 show the fractal dimension variation on time. It can be seen that this parameter rises but reaches a stable point, and this stabilization is achieved faster for the $10.3 \mathrm{~mA}$ experiments. For the first rings, it is observed a higher value of the fractal dimension due to a faster filling effect of the surface, a result mainly linked to nucleation (with a fractal dimension of 2). The value of the fractal dimension will be reduced as the ramifications are more significant, and the diameter of the circles is increased.

By applying this methodology, it is observed that for smaller diameters, this problem will always be present. If the diameter analysis rises as seen in figures 3 and 4, the fractal dimension is reduced. If the circle analyzed presents a big diameter (approximately 900 pixels), the mass-radius fractal dimension (MRFd) would be diminished because of the space inside the crystal (space between ramifications). Therefore, the MRFd shows an apparent stabilization of the fractal dimension for the analysis of the concentrical rings over time. For the final image, Figure 5 shows the fractal dimension results, which indicates an average value of 1.71 as DLA growth for both cases, 3.0 and $10.3 \mathrm{~mA}$.

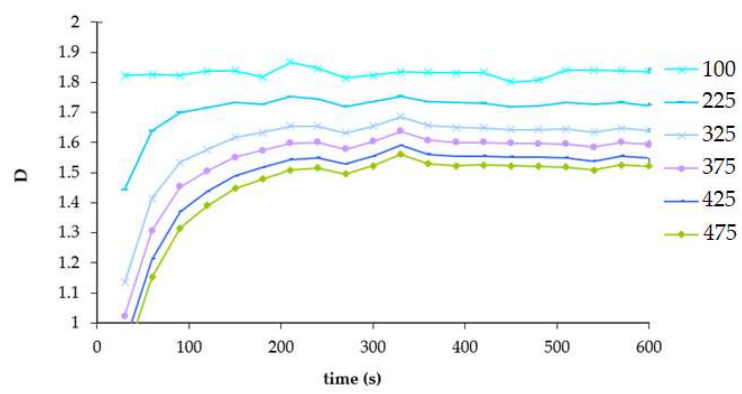

Figure 3: Fractal dimension (D) of concentric rings at $3 \mathrm{~mA}$. Numbers in column (right) correspond to the radium in pixels.

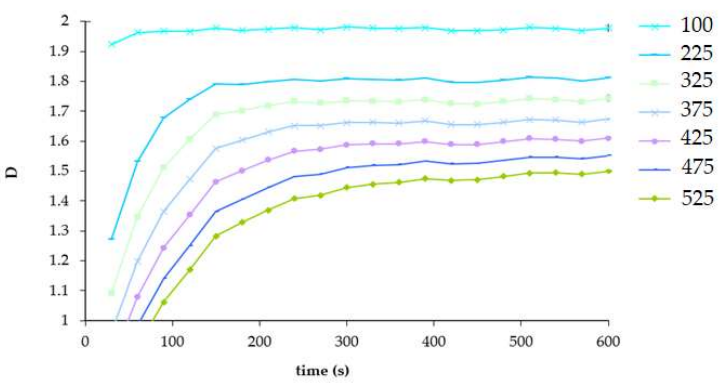

Figure 4: Accumulative fractal dimension (D) of concentrical rings at $10.3 \mathrm{~mA}$. Numbers in column (right) correspond to the radium in pixels

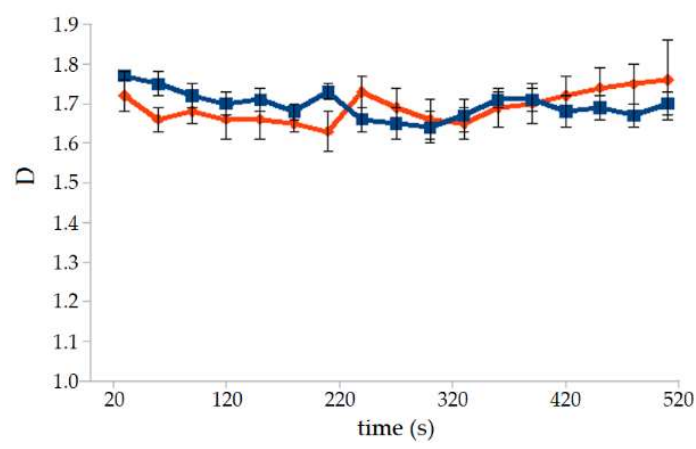

Figure 5: Fractal dimension over time for $10.3 \mathrm{~mA}$ (blue) and 3.0 (red) $(\mathrm{V}=10 \mathrm{~mL}, \mathrm{Ci}=0.1026 \mathrm{~mol} / \mathrm{L}$, and $\mathrm{r}=44 \mathrm{~mm})$ 


\subsection{MRFd Stabilization Model}

The mass of a compound deposited $(m)$ is determined for the equation (1):

$m=k I t$

$I$ is the current (A), $t$ is time (s), and $k$ is an adjustment constant. In the same way, it is easy to determine the mass of the deposit if its density $(\rho)$, radius $(R)$, and fractal dimension $D$ are known:

$m=\rho(R)^{D}$

From equation (1), it can be obtained the variation of mass over time:

$\frac{d m}{d t}=\rho(R)^{D}\left[\frac{D d R}{R d t}+\left(\frac{d D}{d t}\right) \ln R\right]$

By combining equations (1) and (3), it can be deduced the variation of the fractal dimension over time:

$\frac{d D}{d t}=\left[\frac{k I}{\rho(R)^{D}}-\frac{D d R}{R d t}\right] \frac{1}{\ln R}$

From equation (4), it can be obtained:

$\lim _{R \rightarrow \infty} \frac{d D}{d t}=\lim _{R \rightarrow \infty}\left[\frac{k I}{\rho(R)^{D}}-\frac{D d R}{R d t}\right] \frac{1}{\ln R}$

For $R \rightarrow \infty, d R / d t \rightarrow 0$, and $D / R \rightarrow 0$, therefore:

$\lim _{R \rightarrow \infty} \frac{d D}{d t}=\lim _{R \rightarrow \infty}\left[\frac{k I}{\rho(R)^{D}}\right] \frac{1}{\ln R}$

If $D>1$, then $R^{D} \rightarrow \infty$, and $1 / R^{D} \rightarrow 0$. For case $D<1, R^{D} \rightarrow 1$, and $1 / R^{D} \rightarrow 1$. But in any case, if $\mathrm{R} \rightarrow \infty$ then $\ln R \rightarrow \infty$. Then, for $D>1$ :

$\lim _{R \rightarrow \infty} \frac{d D}{d t}=0$

Then, in equation (7), while the radius $R$ grows up, the value of MRFd is going to be stable. This result is in concordance with the experimental behavior observed for 3 and $10.3 \mathrm{~mA}$.

\section{2. Fractal Dimension Predictive Model}

The model was obtained under the next considerations: i) the microscopic entities describing the system are the molecular aggregates on the 2D structure with an annular geometry of radius $R$; ii) the microscopic variable is the number $n$ of entities, for which the transition probabilities overtime for the increment $n+1$ and the decrement $n-1$ are:

$W_{n+1 / n}=k_{1} N_{0}$

$W_{n-1 / n}=k_{2} n$

$k_{1}$ and $k_{2}$ are the velocity constants of the processes, and $N_{0}$ represents the total ions in the solution that in this case is considered constant; iii) the occupied space $\phi$ represents the system evolution for $n$ over the perimetral border $l$ of the total aggregate: 
$\phi=\frac{n l}{R}$

The changes in a microscopic entity for an individual process can be considered null compared with its total number; thus, $n$ is a continuous variable. Therefore, the Fokker-Planck equation (FPE) for the transition probability of $\phi$ is written as:

$\frac{\partial P(\phi ; t)}{\partial t}=-\frac{\partial}{\partial \phi}\left(\frac{l k_{1} N_{0}}{R}-k_{2} \phi\right) P(\phi ; t)+\frac{1}{2} \frac{1}{\Omega} \frac{\partial^{2}}{\partial \phi^{2}}\left(\frac{l k_{1} N_{0}}{R}-k_{2} \phi\right) P(\phi ; t)$

$P\left(\phi_{0} ; 0\right)=1$

Where $1 / \Omega=l / R$. FPE is linear for the transition probabilities per unit of time; then, its solution is a gaussian function where the temporal behavior of the expected value $\Phi=\langle\phi\rangle$ and the variance $\sigma=\left\langle(\phi-\langle\phi\rangle)^{2}\right\rangle$ of the intern fluctuations are described through the differential equations (11) and (12):

$\frac{d \Phi}{d t}=\frac{l k_{1} N_{0}}{R}-k_{2} \Phi$

$\Phi(0)=0$

$\frac{d \sigma}{d t}=-2 k_{2} \sigma+\frac{1}{\Omega}\left(\frac{l k_{1} N_{0}}{R}+k_{2} \Phi\right)$

$\sigma(0)=0$

The exact analytical solutions of equations (11) and (12) are:

$\Phi(t)=\alpha\left(1-e^{-t k_{2}}\right)$

$\sigma(t)=\frac{\alpha}{\Omega}\left(1-e^{-t k_{2}}\right)$

$\alpha=\frac{l N_{0} k_{1}}{R k_{2}}$

$\alpha$ is the condition of the steady-state. The system is studied in a quasi-stationary state, and $\sigma$ is expressed as a function of $\Phi$ :

$\sigma=\frac{1}{2 \Omega}\left(\Phi+\alpha_{q s}\right)$

Where $\alpha_{q s}$ is the expected value in a time $t$. For an ergodic process, the average temporal mid-processes are considered equal to the average of the bulk. Thus, the probability expected value is expressed in terms of density ( $C$ is a proportionality constant):

$\rho=\langle P(\phi)\rangle=\frac{C}{(\sigma)^{\frac{1}{2}}}$

Besides, the variance $\sigma$ can be expressed as a potential function of $\Phi$ :

$\sigma=\Phi^{\mu}$ 
$\mu=\lim _{\Phi \rightarrow 1}\left[\left(\frac{d \ln \left(\frac{1}{2 \Omega}\left(\Phi+\alpha_{q S}\right)\right)}{d \Phi}\right)\left(\frac{d \ln \Phi}{d \Phi}\right)^{-1}\right]=\frac{1}{\alpha_{q s}+1}$

From the fractal dimension concept, the total quantity of substance $\varepsilon$ within a surface $\pi \Phi^{2}$ is:

$\varepsilon=C \Phi^{D}=\rho\left(\pi \Phi^{2}\right)$

After mathematical treatment and substituting equation (13) for the expected value of $\Phi$ in a time $t$ :

$D=2-\frac{1}{\alpha\left(1-e^{-t k_{2}}\right)+1}$

Equation (15) is adjusted as:

$\alpha=\frac{\beta}{R}$

Where $\beta$ depends on the ions concentration and the dynamic parameters that determine the system evolution:

$\beta=l N_{0} \frac{k_{1}}{k_{2}}$

Thereby, for a given system, the fractal dimension $D$ depends on the system expansion over time, in such a way that:

$D=\frac{R+2 \beta-2 \beta e^{-t k_{2}}}{R+\beta-\beta e^{-t k_{2}}}$

Figure 6 shows the predicted values according to equation (24). Equation (24) can be simplified to adjust the experimental data. In this case, the first four terms of the potential series for $t$ are presented in equation (25):

$D=1+A_{1} \frac{t}{R}-A_{2}\left(\frac{t}{R}\right)^{2}-A_{3} \frac{t^{2}}{R}$

In equation (25), the $A_{j}$ constants are obtained for the statistical analysis of the experimental data. The models for the experimental conditions were adjusted through the Marquardt no-linear regression method with the Statgraphics software and are shown in Table 1. Figure 7 is a comparison between the predicted and experimental values.

The values of $\beta$ and $k_{2}$ were calculated from their relation with the constants $A_{1}$ and $A_{3}$ according to the system of equations:

$A_{1}=\beta k_{2}$

$A_{3}=\frac{\beta k_{2}{ }^{2}}{2}$ 


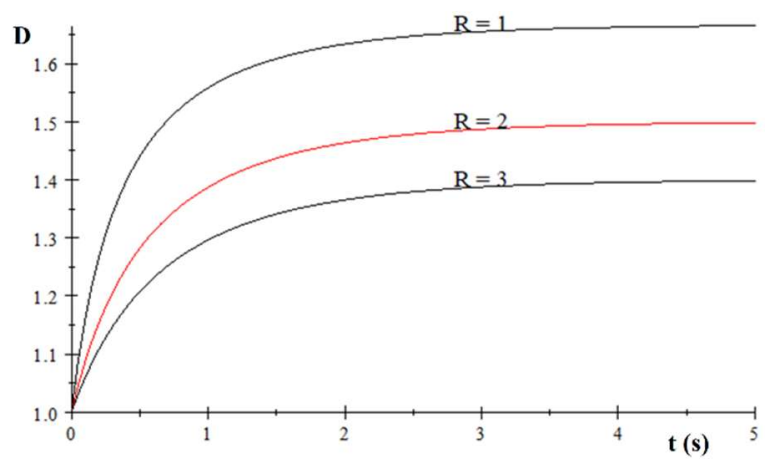

Figure 6: Fractal dimension over time for $R$ as the independent variable in a hypothetical system with $k_{2}=1$, and $\beta$ $=2$.

Table 1: Statistical adjustments of the model for the experimental results

\begin{tabular}{|l|l|l|}
\hline & \multicolumn{1}{|c|}{$\mathbf{I}=\mathbf{1 0 . 3} \mathbf{~ m A}$} & \multicolumn{1}{|c|}{$\mathbf{I}=\mathbf{3} \mathbf{~ m A}$} \\
\hline Adjusted model & $D=1.06+0.65 \frac{t}{R}-0.025\left(\frac{t}{R}\right)^{2}-0.00065 \frac{t^{2}}{R}$ & $D=1.7+0.09 \frac{t}{R}-0.007\left(\frac{t}{R}\right)^{2}-0.0005 \frac{t^{2}}{R}$ \\
\hline $\mathbf{R}^{2}$ & $61.4343 \%$ & $29,4001 \%$ \\
\hline$\beta$ & 323.48 & 67,310 \\
\hline $\boldsymbol{k}_{2}$ & 0.0020 & 0,0010 \\
\hline
\end{tabular}

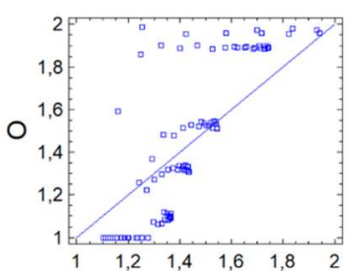

a. $P$

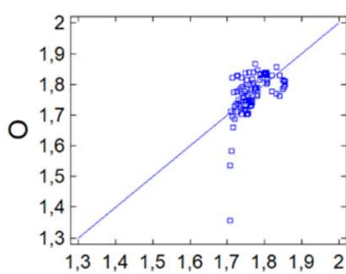

b.

Figure 7: Comparison between the observed $(O)$ and the predicted $(P)$ fractal dimension results according to the adjusted models for each experimental test a) $10.3 \mathrm{~mA}$ and b) $3 \mathrm{~mA}$

\section{CONCLUSIONS}

For the morphological analysis, it was found that the growth achieves a stabilization limit, which follows the mathematical analysis where $\lim _{r \rightarrow \infty} d D / d t=0$ it is applicable for both: rings analysis and mass-radium fractal dimension for the total image. Fractal dimension is 1.71 for the final image, and for the first ring, it decreases to an average value of 1.4, which is expected for a zinc distribution as it zooms out through the branches. The stabilization of the fractal dimension is achieved for $100 \leq \mathrm{R} \leq 375$ pixels. The stochastic model showed a better correlation (61.43\%) for the highest current value, which indicates that the faster deposition velocity produces a more stable fill-up effect of the spaces in the surface.

\section{ACKNOWLEDGEMENTS}

Authors thanks the PROFEXCE program (FADU2020-01) for the analytical equipment support.

\section{REFERENCES}

1. Li, C., Dai, R., Wu, X., Qi, R., and Ma, J., "Electrochemical fabrication of silver dendrites as an excellent platform for surface enhanced Raman Scattering application", International Journal of Electrochemical Science, Vol. 12, (2017), 3252-3262.

2. López-Salvans, M.Q., Sagués F., Claret, J., and Bassas, J., "Fingering instability in thin-layer electrodeposition: General 
trends and morphological transitions", Journal of Electrochemistry, Vol. 421, (1997), 205-212.

3. Trigueros, P.P., Sagués, F., Claret, J., and Mas, F., "Some effects of cell dimensions on Zinc electrodeposits" Journal of Electroanalytical Chemistry, Vol. 328, (1992), 165-178.

4. Seyedraoufi, Z. S., Mirdamadi, S., and Rastegari, S., "Electrodeposition of nano-hydroxyapatite coating on biodegradable Mg-Zn scaffold”, International Journal of Engineering, Transaction C: Aspects, Vol. 27, No. 6, (2014), 939-944.

5. Yousefpour, M., Vali, I., and Saebnoori, E., "Surface activation of Ni-Ti alloy using electrochemical process for biomimetic deposition of hydroxyapatite coating”, International Journal of Engineering, Transaction A: Basics, Vol. 27, No. 10, (2014), 1627-1634.

6. Toghraei, M., and Siadati, H., "Electrodeposited Co-Pi catalyst on $\alpha-\mathrm{Fe}_{2} \mathrm{O}_{3}$ photoanode for water-splitting applications", International Journal of Engineering, Transaction C: Aspects, Vol. 31, No. 12, (2018), 2085-2091.

7. Argoul, F., Arneodo, A., Grasseau, G., and Swinney, H.L., "Self-similarity of diffusion-limited aggregates and electrodeposition clusters", Physical Review Letters, Vol. 61, No. 22, (1988), 2558-2561.

8. Santos, N. M., and Santos, D. M. F., "A fractal dimension minimum in electrodeposited copper dendritic patterns”, Chaos, Solitons \& Fractals, Vol. 116, (2018), 381-385.

9. Tejera, E., Brizuela, N., Rieumont, J., and Nieto-Villar, J.M., "Fractal analysis in epigenetic differentiation of leukemic cells", HarFA-Harmonic and Fractal Image Analysis, (2004), 74-75.

10. Trigueros, P.P., Sagués, F., and Claret, J., "Influence of an inert electrolyte on the morphology of quasi-two-dimensional electrodeposits", Physical Review, Vol. 49, No. 5, (1994), 4328-4335.

11. Gupta, R., Ghosh, S., Choudhury, S., and Ghosh, S., "Pattern transition from dense branching morphology to fractal for copper and $\beta^{\prime}$ brass electrodeposition in thin gap geometry", AIP Advances, Vol. 8, No. 1, (2018), 015219.

12. Argoul, F., Huth, J., Merzeau, P., Arnéodo, A., and Swinney, H.L., "Experimental evidence for homoclinic chaos in an electrochemical growth process", Physica D: Nonlinear Phenomena, Vol. 62, No. 1-4, (1993), 170-185.

13. Sander, L.M., "Diffusion-limited aggregation: a kinetic critical phenomenon?”, Contemporary Physics, Vol. 41, No. 16, (2000), 203-218.

14. Trigueros, P.P., Claret, J., Masc, F., and Sagués, F., "Pattern morphologies in Zinc electrodeposition", Journal of Electroanalytical Chemistry, Vol. 312, (1991), 219-235.

15. Sultan, R., Safieddine, A., Farah, H., and Eddin, A. Z., "Fractal structures in electroless metal deposition systems." In Chaos, Complexity, and Transport: Proceedings of the CCT'15 Conference on Chaos, Complexity and Transport, 2015, 268-282.

16. Suárez-Dominguez, E. J., \& Aranda-Jimenez, Y. G. (2013). Diferencia fractal en superficies de tierra vertida con suelo de Tamaulipas. Contexto. 7(7), 65-72.

17. Trejo-Franco, A. A., Arvizu-Sánchez, E., Brandt-García, L. A., Aranda-Jiménez, Y. G., Lorenzo-Palomera, J. G., \& SuárezDominguez, E. J. (2020). Paint adsorption on walls: I. Fractal-Stochastic model. International Journal, 8(5).

18. Rasband, W.S., ImageJ, U. S. National Institute of Health, Bethesda, Maryland, USA, 1997-2006. http://rsb.info.nih.gov/ij/

19. Suárez-Domínguez, E. J., Brizuela, N., Tejera, E., Quintana, R., Mar, J. A. B., and Nieto-Villar, J. M., "La dimension fractal en la formación de patrones", Revista Cubana de Química, Vol. 18, No. 1, (2006), 311. 


\section{NOMENCLATURE}

\begin{tabular}{|c|c|c|c|}
\hline$A_{1,2,3}$ & $\begin{array}{l}\text { Constants of the adjusted model for } \\
\text { experimental data }\end{array}$ & & ek Symbols \\
\hline$C$ & Proportional constant & $\alpha$ & Condition of the steady-state \\
\hline$D$ & Fractal Dimension & $\beta$ & Subparameter of $\alpha$ \\
\hline$I$ & Current (A) & $\varepsilon$ & Total quantity of substance deposited \\
\hline$k$ & Adjustment constant & $\mu$ & Potential function of $\Phi$ \\
\hline$k_{1,2}$ & $\begin{array}{l}\text { Velocity constants for the system expansion } \\
\text { process }\end{array}$ & $\rho$ & Density \\
\hline$l$ & Length of the fractal perimeter $(\mathrm{m})$ & $\sigma$ & Variance \\
\hline$t$ & Time (s) & $\phi$ & Surface occupied by the entities $n$ \\
\hline$m$ & Mass of a compound deposit (g) & $\Phi$ & Temporal behavior of the expected value of $\phi$ \\
\hline$n$ & $\begin{array}{l}\text { Number of entities in the electrodeposition } \\
\text { process }\end{array}$ & $\Omega$ & Ratio $R / l$ \\
\hline$N_{0}$ & Total ions $\left(\mathrm{Zn}^{+2}\right)$ in the solution & & \\
\hline$R$ & Radius (pixel) & & scripts \\
\hline$W$ & Transition probabilities over time & qs & Quasi-stationary \\
\hline
\end{tabular}



